\title{
Fractional View Analysis of Third Order Kortewege-De Vries Equations, Using a New Analytical Technique
}

\author{
Rasool Shah ${ }^{1 \dagger}$, Umar Farooq ${ }^{1 \dagger}$, Hassan Khan ${ }^{1 \dagger}$, Dumitru Baleanu ${ }^{2,3 \dagger}$, Poom Kumam ${ }^{4,5 * \dagger}$ \\ and Muhammad Arif ${ }^{1 \dagger}$
}

${ }^{1}$ Department of Mathematics, Abdul Wali Khan University Mardan, Mardan, Pakistan, ${ }^{2}$ Department of Mathematics, Faculty of Arts and Sciences, Cankaya University, Ankara, Turkey, ${ }^{3}$ Institute of Space Sciences, Măgurele, Romania, ${ }^{4}$ Center of Excellence in Theoretical and Computational Science (TaCS-COE) and KMUTTFixed Point Research Laboratory, Room SCL 802 Fixed Point Laboratory, Departments of Mathematics, Faculty of Science, King Mongkut's University of Technology Thonburi (KMUTT), Bangkok, Thailand, ${ }^{5}$ Department of Medical Research, China Medical University Hospital, China Medical University, Taichung, Taiwan

\section{OPEN ACCESS}

Edited by:

Ahmed Zeeshan,

International Islamic University,

Islamabad, Pakistan

Reviewed by:

Marin I. Marin

Transilvania University of Braşov,

Romania

Aaqib Majeed,

Bacha Khan University, Pakistan

Ilyas Khan,

Ton Duc Thang University, Vietnam

${ }^{*}$ Correspondence:

Poom Kumam

poom.kum@kmutt.ac.th

tThese authors have contributed equally to this work

Specialty section:

This article was submitted to

Mathematical Physics,

a section of the journal

Frontiers in Physics

Received: 04 September 2019 Accepted: 19 December 2019

Published: 17 January 2020

Citation:

Shah R, Farooq U, Khan H, Baleanu D, Kumam $P$ and Arif $M$ (2020) Fractional View Analysis of Third Order Kortewege-De Vries Equations, Using a New Analytical Technique. Front. Phys. 7:244. doi: 10.3389/fphy.2019.00244
In the present article, fractional view of third order Kortewege-De Vries equations is presented by a sophisticated analytical technique called Mohand decomposition method. The Caputo fractional derivative operator is used to express fractional derivatives, containing in the targeted problems. Some numerical examples are presented to show the effectiveness of the method for both fractional and integer order problems. From the table, it is investigated that the proposed method has the same rate of convergence as compare to homotopy perturbation transform method. The solution graphs have confirmed the best agreement with the exact solutions of the problems and also revealed that if the sequence of fractional-orders is approaches to integer order, then the fractional order solutions of the problems are converge to an integer order solution. Moreover, the proposed method is straight forward and easy to implement and therefore can be used for other non-linear fractional-order partial differential equations.

Keywords: analytical solution, Mohand transform, Adomian decomposition, caputo derivatives, third order Kortewege-De Vries equations

\section{INTRODUCTION}

The class of partial differential equations known as Korteweg-De Vries (KDV) equation which play a vital role in the diverse field of physics such as fluid mechanics, signal processing, hydrology, viscoelasticity and fractional kinetics [1,2]. The KDV equation was first time derived by Korteweg and Vries in 1895. The KDV equation used to model long waves, tides, solitary waves, and wave propagating in a shallow canal. A partial differential Kortewege-De Vries equation of third order is also applied to study the non-linear model of water waves in superficial canal certain namely canal [3], in the time when wave in water was of important concentration in applications in navigational design and also for the awareness of flood and tides [4, 5]. The applications in numerous areas of physics, applied science and other scientific applications, therefore the excessive amount of investigation as a research work has been capitalized in the study of KDV equations [6-10]. We considered the third order time fractional KDV equation in the form [1]

$$
\frac{\partial^{\gamma} u(\chi, \Im)}{\partial \mathfrak{\Im}^{\gamma}}+\kappa u(\chi, \Im) \frac{\partial u(\chi, \Im)}{\partial \chi}+\lambda \frac{\partial^{3} u(\chi, \Im)}{\partial \chi^{3}}+\psi(\chi, \Im), \quad 0<\gamma \leq 1,
$$


with initial source

$$
u(\chi, 0)=u(\chi)
$$

where, $\kappa$ and $\lambda$ are real numbers.

The KdV equations of fractional order can be applied to examine the influence of the higher-order wave dispersion. The $\mathrm{KdV}$-Burgers equation defines the waves on lower water surfaces. The strength of fractional KdV equation is the non-local property [11-21]. For a higher order Korteweg-de Vries equation, which is a natural extension of the Korteweg-de Vries equation written in a bilinear form, a Bcklund conversion in bilinear forms is provided. For this higher-order equation the Bcklund transition is given in ordinary forms and the inverse scattering scheme [22], Korteweg-de Vries type of equations 3rd order coefficient variable [23] and Solution of the third order Korteweg-De Vries homotopy perturbation approach using elzaki transform [24].

In few decades, integral transform of various types such as Fourier transform, Laplace transform, Hankel transform, Mellin transform, Z-transform, Wavelet transform, Elzaki transform, Kamal transform, Mahgoub transform, Aboodh transform, Mohand transform, Sumudu transform, Hermite transform etc., gained a enormous importance in solving advanced model in the field mathematics, physics and engineering [25-36].

In the current article, we have applied the Mohand transform with decomposition procedure for the analytical treatment of time fractional KDV equation. The Mohand Transform is one of the new integral transform use for the analytical treatment of different physical phenomena are molded by Differential Equations (DEs) of integer order or Fractional Partial Differential Equations (FPDEs). Recently, Kumar and Viswanathan used Mohand transform and solved the mechanics and electrical circuit problems [37]. Aggarwal have Comparatively Studied Mohand and Aboodh transforms for the solution of differential equations. The numerical applications reflect that both the transforms (Mohand and Aboodh transforms) are closely related to each other [38]. Sudhanshu Aggarwal have also discussed the comparative study of Mohand and Laplace transforms, Mohand and Sumudu transforms, Mohand and Mahgoub transforms [39-41]. Sudhanshu Aggarwal have successfully discussed the Mohand transform of Bessels functions of zero, one and two orders, which is very useful for solving many equations in cylindrical or spherical coordinates such as heat equation, wave equation etc. [42]. The exact solution of second kinds of linear Volterra integral equations get by using Mohand transform. It is claimed that Mohand transform take very little time and has no large computational work [43]. Mohand transform have also used the for solution of Abel's integral equation. The obtained results show that Mohand transform is a powerful integral transform for handling Abel's integral equation [44]. The remaining section of the paper are managed as follows. In the second section, we present some related definitions of fractional calculus and basic concepts of Mohand transform. The third section presents the implementation the proposed methodology. The four section represent different models of $\mathrm{KDV}$ equation are examined separately and plotted. Finally, we depict our conclusions.

\section{PRELIMINARIES CONCEPTS}

In this section, we present some basic necessary definitions and preliminaries concepts related to fractional calculus and Mohand transform.

\section{DEFINITION}

Mohand transform first time was define by Mohand and Mahgoub of the function $u(\Im)$ for $\mathfrak{s} \geq 0$ in the year 2017. The Mohand transform which is represented by $M($.$) for a function$ $u(\tau)$ is define as [45]

$$
M\{u(\Im)\}=R(v)=v^{2} \int_{0}^{\infty} u(\Im) e^{-v \Im} d \Im, \quad k_{1} \leq v \leq k_{2},
$$

The Mohand transform of a function $u(\Im)$ is $R(v)$ then $u(\Im)$ is called the inverse of $R(v)$ which is expressed as.

$$
M^{-1}\{R(v)\}=u(\Im), M^{-1} \text { is inverse Mohand operator. }
$$

\section{DEFINITION}

Mohand transform for $n$th derivatives [46]

$M\left\{u^{n}(\Im)\right\}=v^{n} R(v)-v^{n+1} u(0)-v^{n} u^{\prime}(0)-\cdots-v^{2} u^{n-1}(0)$,

\section{DEFINITION}

Mohand transform for fractional order derivatives [46]

$$
R\left\{u^{\gamma}(\Im)\right\}=v^{\gamma} R(v)-\sum_{k=0}^{n-1} \frac{u^{k}(0)}{v^{k-(\gamma+1)}}, \quad 0<\gamma \leq n,
$$

\section{DEFINITION}

Caputo operator of fractional partial derivative [47]

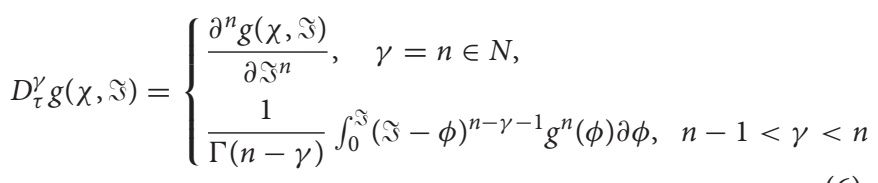

\section{IMPLEMENTATION OF MOHAND TRANSFORM}

In this section we have considered the time fractional KDV model in the form

$$
\begin{aligned}
& \frac{\partial^{\gamma} u(\chi, \Im)}{\partial \Im^{\gamma}}+\kappa u(\chi, \Im) \frac{\partial u(\chi, \Im)}{\partial \chi}+\lambda \frac{\partial^{3} u(\chi, \Im)}{\partial \chi^{3}}=\psi(\chi, \Im) \\
& \quad 0<\gamma \leq 1,
\end{aligned}
$$

with initial source

$$
u(\chi, 0)=u(\chi) .
$$

where, $\kappa$ and $\lambda$ are real numbers. 
Applying Mohand transform [45]

$$
\begin{aligned}
M\left\{\frac{\partial^{\gamma} u(\chi, \Im)}{\partial \Im^{\gamma}}\right. & \left.+\kappa u(\chi, \Im) \frac{\partial u(\chi, \Im)}{\partial \chi}+\lambda \frac{\partial^{3} u(\chi, \Im)}{\partial \chi^{3}}\right\} \\
& =M\{\psi(\chi, \Im)\}, 0<\gamma \leq 1,
\end{aligned}
$$

by using the transform property, we can simplify as

$$
\begin{aligned}
v^{\gamma}\{R(v)-v u(0)\} & +M\left\{\kappa u(\chi, \Im) \frac{\partial u(\chi, \Im)}{\partial \chi}+\lambda \frac{\partial^{3} u(x, \tau)}{\partial x^{3}}\right\} \\
& =M\{\psi(x, \tau)\},
\end{aligned}
$$

after some evaluation, Equation (8) simplified as

$$
\begin{aligned}
R(v)= & v u(0)+\frac{1}{v^{\gamma}} M\left\{-\kappa u(x, \tau) \frac{\partial u(x, \tau)}{\partial x}-\lambda \frac{\partial^{3} u(\chi, \Im)}{\partial \chi^{3}}\right\} \\
& +\frac{1}{v^{\gamma}} M\{\psi(\chi, \Im)\},
\end{aligned}
$$

by applying inverse Mohand transform

$$
\begin{aligned}
u(\chi, \Im)= & v u(0)+M^{-1}\left\{\frac { 1 } { v ^ { \gamma } } M \left\{-\kappa u(\chi, \Im) \frac{\partial u(\chi, \Im)}{\partial \chi}\right.\right. \\
& \left.\left.-\lambda \frac{\partial^{3} u(\chi, \Im)}{\partial \chi^{3}}\right\}+\frac{1}{v^{\gamma}} M\{\psi(\chi, \Im)\}\right\} .
\end{aligned}
$$

Finally we obtain the recursive general relation as

$$
\begin{aligned}
u_{0}(\chi, \Im)= & u(0)+M^{-1}\left\{\frac{1}{v^{\gamma}} M\{\psi(\chi, \Im)\}\right\} \quad m=0 \\
u_{m+1}(\chi, \Im)= & M^{-1}\left\{\frac { 1 } { v ^ { \gamma } } M \left\{-\kappa u_{m}(\chi, \Im) \frac{\partial u_{m}(\chi, \Im)}{\partial \chi}\right.\right. \\
& \left.\left.-\lambda \frac{\partial^{3} u_{m}(\chi, \Im)}{\partial \chi^{3}}\right\}\right\}, \quad m \geq 0 .
\end{aligned}
$$

\section{THEOREM}

Let $\chi$ and $\mathcal{Y}$ be two Banach spaces and $T: \chi \rightarrow \mathcal{Y}$ be a contractive nonlinear operator, such that for all $u ; u^{*} \in ; \chi,\left\|T(u)-T\left(u^{*}\right)\right\| \leq$ $K\left\|u-u^{*}\right\|, \quad 0<K<1[48]$.

Then, in view of Banach contraction theorem, $T$ has a unique fixed point $u$, such that $T u=u$ : Let us write the generated series (12), by the Mohand decomposition method as

$$
\chi_{m}=T\left(\chi_{m-1}\right), \quad \chi_{m-1}=\sum_{j=1}^{m-1} u_{j}, \quad j=0,1,2, \cdots
$$

and supposed that $\chi_{0}=u_{0} \in \mathcal{S}_{p}(u)$, where $\mathcal{S}_{p}(u)=\left\{u^{*} \in\right.$ $\left.\chi:\left\|u-u^{*}\right\|<p\right\}$ then, we have

$$
\begin{aligned}
& \left(B_{1}\right) \chi_{m} \in \mathcal{S}_{p}(u) \\
& \left(B_{2}\right) \lim _{m \rightarrow \infty} \chi_{n}=u .
\end{aligned}
$$

\section{Proof}

$\left(B_{1}\right)$ In view of mathematical induction for $m=1$, we have

$$
\left\|\chi_{1}-u_{1}\right\|=\left\|T\left(\chi_{0}-T(u)\right)\right\| \leq K\left\|u_{0}-u\right\| .
$$

Let the result is true for $m-1$, then

$$
\left\|\chi_{m-1}-u\right\| \leq K^{m-1}\left\|u_{0}-u\right\| .
$$

We have

$\left\|\chi_{m}-u\right\|=\left\|T\left(\chi_{m-1}-T(u)\right)\right\| \leq K\left\|\chi_{m-1}-u\right\| \leq K^{m}\left\|u_{0}-u\right\|$.

Hence, using $\left(B_{1}\right)$, we have

$$
\left\|\chi_{m}-u\right\| \leq K^{m}\left\|u_{0}-u\right\| \leq K^{m} p<p
$$

which implies that $\chi_{m} \in \mathcal{S}_{p}(u)$.

$\left(B_{2}\right)$ : Since $\left\|\chi_{m}-u\right\| \leq K^{m}\left\|u_{0}-u\right\|$ and as a $\lim _{m \rightarrow \infty}$ $K^{m}=0$.

Therefore; we have $\lim _{m \rightarrow \infty}\left\|u_{n}-u\right\|=0 \Rightarrow \lim _{m \rightarrow \infty}$ $u_{n}=u$.

\section{APPLICATIONS AND DISCUSSION}

Here, we have implemented the Mohand transform on some time fractional KVD equations.

Example 4.1: Consider the third order time fractional KVD equation [49]

$$
\frac{\partial^{\gamma} u(\chi, \Im)}{\partial \Im^{\gamma}}+6 u(\chi, \Im) \frac{\partial u(\chi, \Im)}{\partial \chi}+\frac{\partial^{3} u(\chi, \Im)}{\partial \chi^{3}}=0, \quad 0<\gamma \leq 1,
$$

with initial source

$$
u(\chi, 0)=\chi .
$$

Taking Mohand transform of Equation (12), we get

$$
v^{\gamma}\{R(v)-v u(0)\}=M\left\{-6 u(\chi, \mathfrak{s}) \frac{\partial u(\chi, \Im)}{\partial \chi}-\frac{\partial^{3} u(\chi, \Im)}{\partial \chi^{3}}\right\},
$$

after some evaluation, Equation (13) is simplified as

$$
R(v)=v u(0)+\frac{1}{v^{\gamma}}\left\{M\left\{-6 u(\chi, \Im) \frac{\partial u(\chi, \Im)}{\partial \chi}-\frac{\partial^{3} u(\chi, \Im)}{\partial \chi^{3}}\right\}\right\},
$$

by applying inverse Mohand transform, we get

$u(\chi, \Im)=u(0)+M^{-1}\left\{\frac{1}{v^{\gamma}} M\left\{-6 u(\chi, \Im) \frac{\partial u(\chi, \Im)}{\partial \chi}-\frac{\partial^{3} u(\chi, \Im)}{\partial \chi^{3}}\right\}\right\}$,

thus, by using recursive scheme of Equation (11), we get 


$$
\begin{aligned}
u_{0}(\chi, \Im)= & u(0)=\chi \\
u_{m+1}(\chi, \Im)= & M^{-1}\left\{\frac { 1 } { v ^ { \gamma } } M \left\{-6 u_{m}(\chi, \Im) \frac{\partial u_{m}(\chi, \Im)}{\partial \chi}\right.\right. \\
& \left.\left.-\frac{\partial^{3} u_{m}(\chi, \Im)}{\partial \chi^{3}}\right\}\right\}, \quad m=0,1, \cdots
\end{aligned}
$$

From the recursive formula (17),

$$
\text { for } m=0
$$

$$
\begin{aligned}
& u_{1}(\chi, \Im)=M^{-1}\left\{\frac{1}{v^{\gamma}} M\left\{-6 u_{0}(\chi, \Im) \frac{\partial u_{0}(\chi, \Im)}{\partial \chi}-\frac{\partial^{3} u_{0}(\chi, \Im)}{\partial \chi^{3}}\right\}\right\}, \\
& u_{1}(\chi, \Im)=-6 \chi \frac{\Im^{\gamma}}{\gamma !}
\end{aligned}
$$

for $m=1$

$$
\begin{aligned}
u_{2}(\chi, \Im)= & M^{-1}\left\{\frac { 1 } { v ^ { \gamma } } M \left\{-6 u_{0}(\chi, \Im) \frac{\partial u_{1}(\chi, \Im)}{\partial \chi}\right.\right. \\
& \left.\left.-6 u_{1}(\chi, \Im) \frac{\partial u_{0}(\chi, \Im)}{\partial \chi}-\frac{\partial^{3} u_{1}(\chi, \Im)}{\partial \chi^{3}}\right\}\right\}, \\
u_{2}(\chi, \Im)= & 72 \chi \frac{\Im^{2 \gamma}}{(2 \gamma) !},
\end{aligned}
$$

for $m=2$

$$
\begin{aligned}
u_{3}(\chi, \Im)= & M^{-1}\left\{\frac { 1 } { v ^ { \gamma } } M \left\{-6 u_{0}(\chi, \Im) \frac{\partial u_{2}(\chi, \Im)}{\partial \chi}\right.\right. \\
& -6 u_{1}(\chi, \Im) \frac{\partial u_{1}(\chi, \Im)}{\partial \chi}-6 u_{2}(\chi, \Im) \frac{\partial u_{0}(\chi, \Im)}{\partial \chi} \\
& \left.\left.-\frac{\partial^{3} u_{2}(\chi, \Im)}{\partial \chi^{3}}\right\}\right\}, \\
u_{3}(\chi, \Im)= & -864 \chi \frac{\Im^{3 \gamma}}{(3 \gamma) !}-216 \chi(2 \gamma) ! \frac{\Im^{3 \gamma}}{(3 \gamma) ! \gamma ! \gamma !},
\end{aligned}
$$

Similarly for $m=3$, we can get

$$
\begin{aligned}
u_{4}(\chi, \Im)= & 10368 \chi \frac{\Im^{4 \gamma}}{(4 \gamma) !}+2592 \chi(2 \gamma) ! \frac{\Im^{4 \gamma}}{(4 \gamma) ! \gamma !} \\
& +5184 \chi(3 \gamma) ! \frac{\Im^{4 \gamma}}{(4 \gamma) !(2 \gamma) ! \gamma !},
\end{aligned}
$$

The Mohand transform solution for example 4.1 is

$$
\begin{aligned}
u(\chi, \Im)= & u_{0}(\chi, \Im)+u_{1}(\chi, \Im)+u_{2}(\chi, \Im)+u_{3}(\chi, \Im) \\
& +u_{4}(\chi, \Im)+\cdots \\
u(\chi, \Im)= & \chi-6 x \frac{\Im^{\gamma}}{\gamma !}+72 \chi \frac{\Im^{2 \gamma}}{(2 \gamma) !}-864 \chi \frac{\Im^{3 \gamma}}{(3 \gamma) !} \\
& -216 \chi(2 \gamma) ! \frac{\Im^{3 \gamma}}{(3 \gamma) ! \gamma ! \gamma !}+10368 \chi \frac{\Im^{4 \gamma}}{(4 \gamma) !} \\
& +2592 \chi(2 \gamma) ! \frac{\Im^{4 \gamma}}{(4 \gamma) ! \gamma !}+5184 \chi(3 \gamma) ! \frac{\Im^{4 \gamma}}{(4 \gamma) !(2 \gamma) ! \gamma !}+\cdots
\end{aligned}
$$

For particular case $\gamma=1$, the Mohand transform solution become as

$$
u(x, \tau)=\chi\left(1-6 \Im+36 \Im^{2}-216 \Im^{3}+1296 \Im^{4}+\cdots\right) .
$$

The calculated result provide the exact solution in the close form

$$
u(\chi, \Im)=\frac{\chi}{1+6 \Im} .
$$

Example 4.2: Consider the third order time fractional KVD equation [50]

$$
\frac{\partial^{\gamma} u(\chi, \Im)}{\partial \Im^{\gamma}}+u(\chi, \Im) \frac{\partial u(\chi, \Im)}{\partial \chi}+\frac{\partial^{3} u(\chi, \Im)}{\partial \chi^{3}}=0, \quad 0<\gamma \leq 1,
$$

with initial source

$$
u(\chi, 0)=1-\chi .
$$

Taking Mohand transform of Equation (26)

$$
v^{\gamma}\{R(v)-v u(0)\}=M\left\{-u(\chi, \Im) \frac{\partial u(\chi, \Im)}{\partial \chi}-\frac{\partial^{3} u(\chi, \Im)}{\partial \chi^{3}}\right\},
$$

after some evaluation, Equation (27) is simplified as

$$
R(v)=v u(0)+\frac{1}{v^{\gamma}}\left\{M\left\{-u(\chi, \Im) \frac{\partial u(\chi, \Im)}{\partial \chi}-\frac{\partial^{3} u(\chi, \Im)}{\partial \chi^{3}}\right\}\right\}
$$

taking inverse Mohand transform of Equation (28)

$u(\chi, \Im)=u(0)+M^{-1}\left\{\frac{1}{v^{\gamma}} M\left\{-u(\chi, \Im) \frac{\partial u(\chi, \Im)}{\partial \chi}-\frac{\partial^{3} u(\chi, \Im)}{\partial \chi^{3}}\right\}\right\}$,

by using the recursive scheme Equation (11), we get

$$
\begin{aligned}
u_{0}(\chi, \Im)= & u(0)=1-\chi, \\
u_{m+1}(\chi, \Im)= & M^{-1}\left\{\frac { 1 } { v ^ { \gamma } } M \left\{-u_{m}(\chi, \Im) \frac{\partial u_{m}(\chi, \Im)}{\partial \chi}\right.\right. \\
& \left.\left.-\frac{\partial^{3} u_{m}(\chi, \Im)}{\partial \chi^{3}}\right\}\right\},
\end{aligned}
$$

From the recursive formula (31),

for $m=0$

$$
\begin{aligned}
& u_{1}(\chi, \Im)=M^{-1}\left\{\frac{1}{v^{\gamma}} M\left\{-u_{0}(\chi, \Im) \frac{\partial u_{0}(\chi, \Im)}{\partial \chi}-\frac{\partial^{3} u_{0}(\chi, \Im)}{\partial \chi^{3}}\right\}\right\}, \\
& u_{1}(\chi, \Im)=(1-\chi) \frac{\Im^{\gamma}}{\gamma !}
\end{aligned}
$$

for $m=1$

$$
\begin{aligned}
u_{2}(\chi, \Im)= & M^{-1}\left\{\frac { 1 } { v ^ { \gamma } } M \left\{-u_{0}(\chi, \Im) \frac{\partial u_{1}(\chi, \Im)}{\partial \chi}\right.\right. \\
& \left.\left.-u_{1}(\chi, \Im) \frac{\partial u_{0}(\chi, \Im)}{\partial \chi}-\frac{\partial^{3} u_{1}(\chi, \Im)}{\partial \chi^{3}}\right\}\right\},
\end{aligned}
$$




$$
u_{2}(\chi, \Im)=2(1-\chi) \frac{\Im^{2 \gamma}}{(2 \gamma) !}
$$

for $m=2$

$$
\begin{aligned}
u_{3}(\chi, \Im)= & M^{-1}\left\{\frac { 1 } { v ^ { \gamma } } M \left\{-u_{0}(\chi, \Im) \frac{\partial u_{2}(\chi, \Im)}{\partial \chi}\right.\right. \\
& -u_{1}(\chi, \Im) \frac{\partial u_{1}(\chi, \Im)}{\partial \chi}-u_{2}(\chi, \Im) \frac{\partial u_{0}(\chi, \Im)}{\partial \chi} \\
& \left.\left.-\frac{\partial^{3} u_{2}(\chi, \Im)}{\partial \chi^{3}}\right\}\right\}, \\
u_{3}(\chi, \Im)= & 6(1-\chi) \frac{\Im^{3 \gamma}}{(3 \gamma) !} .
\end{aligned}
$$

The Mohand transform solution for example 3.2 is

$$
\begin{aligned}
u(\chi, \Im)= & u_{0}(\chi, \Im)+u_{1}(\chi, \Im)+u_{2}(\chi, \Im)+u_{3}(\chi, \Im)+. . \\
u(\chi, \Im)= & 1-\chi+(1-\chi) \frac{\Im^{\gamma}}{\gamma !}+2(1-\chi) \frac{\Im^{2 \gamma}}{(2 \gamma) !} \\
& +6(1-\chi) \frac{\Im^{3 \gamma}}{(3 \gamma) !}+\cdots
\end{aligned}
$$

For particular case $\gamma=1$, the Mohand transform solution become as

$$
u(\chi, \Im)=1-\chi\left(1+\Im+\Im^{2}+\Im^{3}+\cdots\right) .
$$

The calculated result provide the exact solution in the close form

$$
u(\chi, \Im)=\frac{1-\chi}{1-\mathfrak{s}}
$$

Example 4.3 Consider the third order time fractional KVD equation [6]

$\frac{\partial^{\gamma} u(\chi, \Im)}{\partial \Im^{\gamma}}-6 u(\chi, \Im) \frac{\partial u(\chi, \Im)}{\partial \chi}+\frac{\partial^{3} u(\chi, \Im)}{\partial \chi^{3}}=0, \quad 0<\gamma \leq 1$,

with initial source

$$
u(\chi, 0)=6 \chi .
$$

Taking Mohand transform of Equation (39)

$$
v^{\gamma}\{R(v)-v u(0)\}=M\left\{6 u(\chi, \Im) \frac{\partial u(\chi, \Im)}{\partial \chi}-\frac{\partial^{3} u(\chi, \Im)}{\partial \chi^{3}}\right\},
$$

after some evaluation, Equation (40) is simplified as

$$
R(v)=v u(0)+\frac{1}{v^{\gamma}}\left\{M\left\{6 u(\chi, \Im) \frac{\partial u(\chi, \Im)}{\partial \chi}-\frac{\partial^{3} u(\chi, \Im)}{\partial \chi^{3}}\right\}\right\},
$$

by applying inverse Mohand transform, we get

$$
u(\chi, \Im)=u(0)+M^{-1}\left\{\frac{1}{v^{\gamma}} M\left\{6 u(\chi, \Im) \frac{\partial u(\chi, \Im)}{\partial \chi}-\frac{\partial^{3} u(\chi, \Im)}{\partial \chi^{3}}\right\}\right\}
$$

thus, by using recursive scheme of Equation (11), we get

$$
\begin{aligned}
u_{0}(\chi, \Im)= & u(0)=6 \chi \\
u_{m+1}(\chi, \Im)= & M^{-1}\left\{\frac { 1 } { v ^ { \gamma } } M \left\{6 u_{m}(\chi, \Im) \frac{\partial u_{m}(\chi, \Im)}{\partial \chi}\right.\right. \\
& \left.\left.-\frac{\partial^{3} u_{m}(\chi, \Im)}{\partial \chi^{3}}\right\}\right\}, \quad m=0,1, \cdots .
\end{aligned}
$$

From the recursive formula (44),

for $m=0$

$$
\begin{aligned}
& u_{1}(\chi, \Im)=M^{-1}\left\{\frac{1}{v^{\gamma}} M\left\{6 u_{0}(\chi, \Im) \frac{\partial u_{0}(\chi, \Im)}{\partial \chi}-\frac{\partial^{3} u_{0}(\chi, \Im)}{\partial \chi^{3}}\right\}\right\}, \\
& u_{1}(\chi, \Im)=216 \chi \frac{\Im^{\gamma}}{\gamma !}, \\
& \text { for } m=1
\end{aligned}
$$

$$
\begin{aligned}
u_{2}(\chi, \Im)= & M^{-1}\left\{\frac { 1 } { v ^ { \gamma } } M \left\{6 u_{0}(\chi, \Im) \frac{\partial u_{1}(\chi, \Im)}{\partial \chi}\right.\right. \\
& \left.\left.+6 u_{1}(\chi, \Im) \frac{\partial u_{0}(\chi, \Im)}{\partial \chi}-\frac{\partial^{3} u_{1}(\chi, \Im)}{\partial \chi^{3}}\right\}\right\}, \\
u_{2}(\chi, \Im)= & 15552 \chi \frac{\Im^{2 \gamma}}{(2 \gamma) !}
\end{aligned}
$$

for $m=2$

$$
\begin{aligned}
u_{3}(\chi, \Im)= & M^{-1}\left\{\frac { 1 } { v ^ { \gamma } } M \left\{6 u_{0}(\chi, \Im) \frac{\partial u_{2}(\chi, \Im)}{\partial \chi}+6 u_{1}(\chi, \Im) \frac{\partial u_{1}(\chi, \Im)}{\partial \chi}\right.\right. \\
& \left.\left.+6 u_{2}(\chi, \Im) \frac{\partial u_{0}(\chi, \Im)}{\partial \chi}-\frac{\partial^{3} u_{2}(\chi, \Im)}{\partial \chi^{3}}\right\}\right\} \\
u_{3}(\chi, \Im)= & 1119744 \chi \frac{\Im^{3 \gamma}}{(3 \gamma) !}+279936 \chi(2 \gamma) ! \frac{\Im^{3 \gamma}}{(3 \gamma) ! \gamma ! \gamma !}
\end{aligned}
$$

The Mohand transform solution for example 4.3 is

$$
\begin{aligned}
u(\chi, \Im)= & u_{0}(\chi, \Im)+u_{1}(\chi, \Im)+u_{2}(\chi, \Im)+u_{3}(\chi, \Im)+\cdots \\
u(\chi, \Im)= & 6 \chi+216 \chi \frac{\Im^{\gamma}}{\gamma !}+15552 \chi \frac{\Im^{2 \gamma}}{(2 \gamma) !}+ \\
& 1119744 \chi \frac{\Im^{3 \gamma}}{(3 \gamma) !}+279936 \chi(2 \gamma) ! \frac{\Im^{3 \gamma}}{(3 \gamma) ! \gamma ! \gamma !}+\cdots
\end{aligned}
$$

For particular case $\gamma=1$, the Mohand transform solution become as

$$
u(\chi, \mathfrak{s})=6 \chi\left(1+36 \mathfrak{s}+1296 \mathfrak{\Im}^{2}+46656 \mathfrak{\Im}^{3}+\cdots\right) .
$$


The calculated result provide the exact solution in the close form

$$
u(\chi, \Im)=\frac{6 \chi}{1-36 \Im} .
$$

Example 4.4 Consider the third order time fractional KVD equation [6]

$\frac{\partial^{\gamma} u(\chi, \Im)}{\partial \Im^{\gamma}}-6 u(\chi, \Im) \frac{\partial u(\chi, \Im)}{\partial \chi}+\frac{\partial^{3} u(\chi, \Im)}{\partial \chi^{3}}=0, \quad 0<\gamma \leq 1$,

with initial source

$$
u(\chi, 0)=\frac{6}{\chi^{2}} .
$$

Taking Mohand transform of Equation (52)

$$
v^{\gamma}\{R(v)-v u(0)\}=M\left\{6 u(\chi, \Im) \frac{\partial u(\chi, \Im)}{\partial \chi}-\frac{\partial^{3} u(\chi, \Im)}{\partial \chi^{3}}\right\},
$$

after some evaluation, Equation (53) is simplified as

$$
R(v)=v u(0)+\frac{1}{v^{\gamma}}\left\{M\left\{6 u(\chi, \Im) \frac{\partial u(\chi, \Im)}{\partial \chi}-\frac{\partial^{3} u(\chi, \Im)}{\partial \chi^{3}}\right\}\right\},
$$

by applying inverse Mohand transform, we get

$u(\chi, \Im)=u(0)+M^{-1}\left\{\frac{1}{v^{\gamma}} M\left\{6 u(\chi, \Im) \frac{\partial u(\chi, \Im)}{\partial \chi}-\frac{\partial^{3} u(\chi, \Im)}{\partial \chi^{3}}\right\}\right\}$.

Thus, by using recursive scheme of Equation (11), we get

$$
\begin{aligned}
u_{0}(\chi, \Im)= & u(0)=\frac{6}{\chi^{2}}, \\
u_{m+1}(\chi, \Im)= & M^{-1}\left\{\frac { 1 } { v ^ { \gamma } } M \left\{6 u_{m}(\chi, \Im) \frac{\partial u_{m}(\chi, \Im)}{\partial \chi}\right.\right. \\
& \left.\left.-\frac{\partial^{3} u_{m}(\chi, \Im)}{\partial \chi^{3}}\right\}\right\}, \quad m=0,1, \cdots .
\end{aligned}
$$
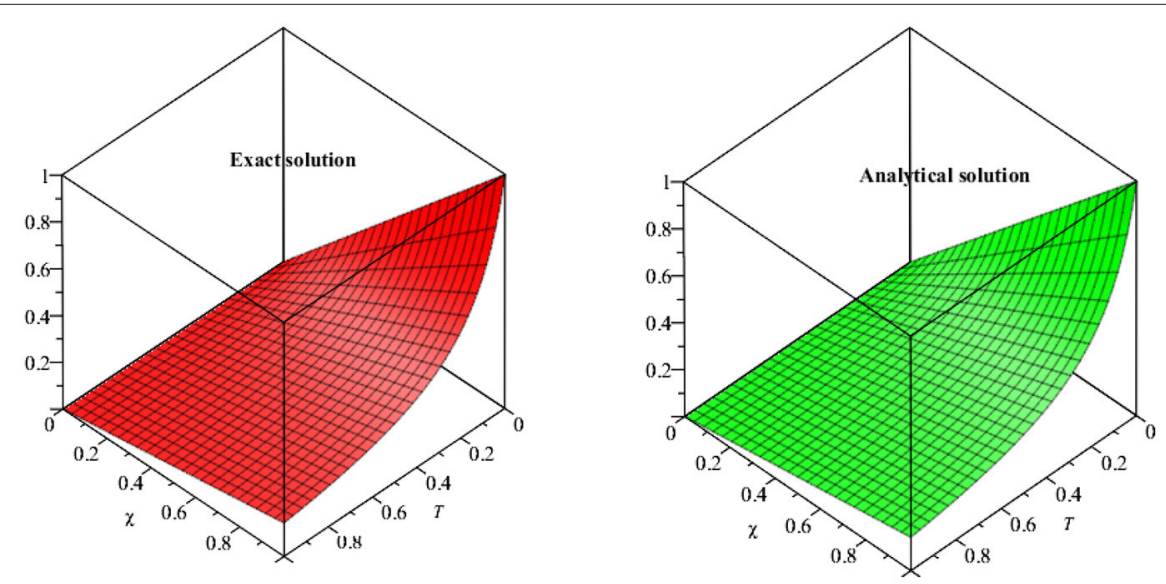

FIGURE 1 | Represents the exact and analytical solution of example 4.1.
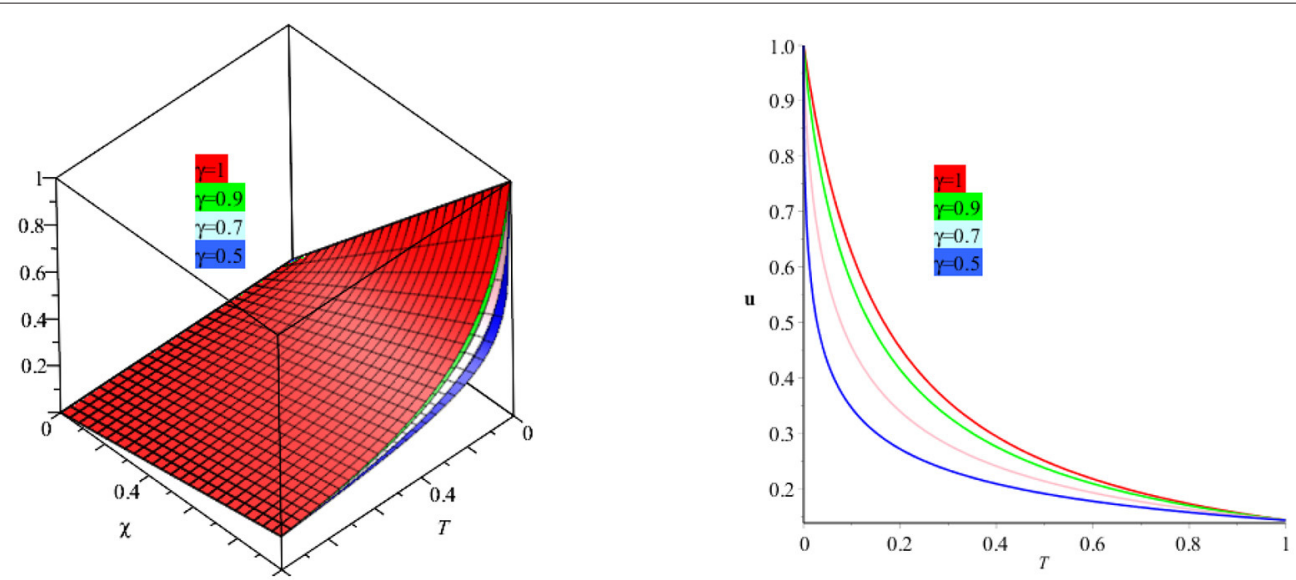

FIGURE 2 | Represents the solution at different fractional order. 
From the recursive formula (44),

for $m=0$

$$
\begin{aligned}
& u_{1}(\chi, \Im)=M^{-1}\left\{\frac{1}{v^{\gamma}} M\left\{6 u_{0}(\chi, \Im) \frac{\partial u_{0}(\chi, \Im)}{\partial \chi}-\frac{\partial^{3} u_{0}(\chi, \Im)}{\partial \chi^{3}}\right\}\right\} \\
& u_{1}(\chi, \Im)=\frac{-288}{\chi^{5}} \frac{\Im^{\gamma}}{\gamma !}
\end{aligned}
$$

for $m=1$

$$
\begin{aligned}
u_{2}(\chi, \Im)= & M^{-1}\left\{\frac { 1 } { v ^ { \gamma } } M \left\{6 u_{0}(\chi, \Im) \frac{\partial u_{1}(\chi, \Im)}{\partial \chi}\right.\right. \\
& \left.\left.+6 u_{1}(\chi, \Im) \frac{\partial u_{0}(\chi, \Im)}{\partial \chi}-\frac{\partial^{3} u_{1}(\chi, \Im)}{\partial \chi^{3}}\right\}\right\}, \\
u_{2}(\chi, \Im)= & \frac{12096}{\chi^{8}} \frac{\Im^{2 \gamma}}{(2 \gamma) !},
\end{aligned}
$$

for $m=2$

$$
\begin{aligned}
u_{3}(\chi, \Im)= & M^{-1}\left\{\frac { 1 } { v ^ { \gamma } } M \left\{6 u_{0}(\chi, \Im) \frac{\partial u_{2}(\chi, \Im)}{\partial \chi}\right.\right. \\
& +6 u_{1}(\chi, \Im) \frac{\partial u_{1}(\chi, \Im)}{\partial \chi}+6 u_{2}(\chi, \Im) \frac{\partial u_{0}(\chi, \Im)}{\partial \chi} \\
& \left.\left.-\frac{\partial^{3} u_{2}(\chi, \Im)}{\partial \chi^{3}}\right\}\right\},
\end{aligned}
$$

$$
u_{3}(\chi, \Im)=\frac{4354560}{\chi^{11}} \frac{\Im^{3 \gamma}}{(3 \gamma) !}-\frac{2488320}{\chi^{11}}(2 \gamma) ! \frac{\Im^{3 \gamma}}{(3 \gamma) ! \gamma ! \gamma !},
$$

The Mohand transform solution for example 4.3 is

$$
\begin{aligned}
u(\chi, \Im)= & u_{0}(\chi, \Im)+u_{1}(\chi, \Im)+u_{2}(\chi, \Im)+u_{3}(\chi, \Im)+. . \\
u(\chi, \Im)= & \frac{6}{\chi^{2}}-\frac{288}{\chi^{5}} \frac{\Im^{\gamma}}{\gamma !}+\frac{12096}{\chi^{8}} \frac{\Im^{2 \gamma}}{(2 \gamma) !}+\frac{4354560}{\chi^{11}} \frac{\Im^{3 \gamma}}{(3 \gamma) !} \\
& -\frac{2488320}{\chi^{11}}(2 \gamma) ! \frac{\Im^{3 \gamma}}{(3 \gamma) ! \gamma ! \gamma !} .
\end{aligned}
$$

For particular case $\gamma=1$, the Mohand transform solution become as

$$
u(\chi, \Im)=\frac{6}{\chi^{2}}-\frac{288}{\chi^{5}} \Im+\frac{6048}{\chi^{8}} \Im^{2}-\frac{103680}{\chi^{11}} \Im^{3}+\cdots
$$

The calculated result converge to the exact solution in the close form

$$
u(\chi, \Im)=\frac{6 \chi\left(\chi^{3}-24 \Im\right)}{\left(\chi^{3}+12 \Im\right)^{2}}
$$
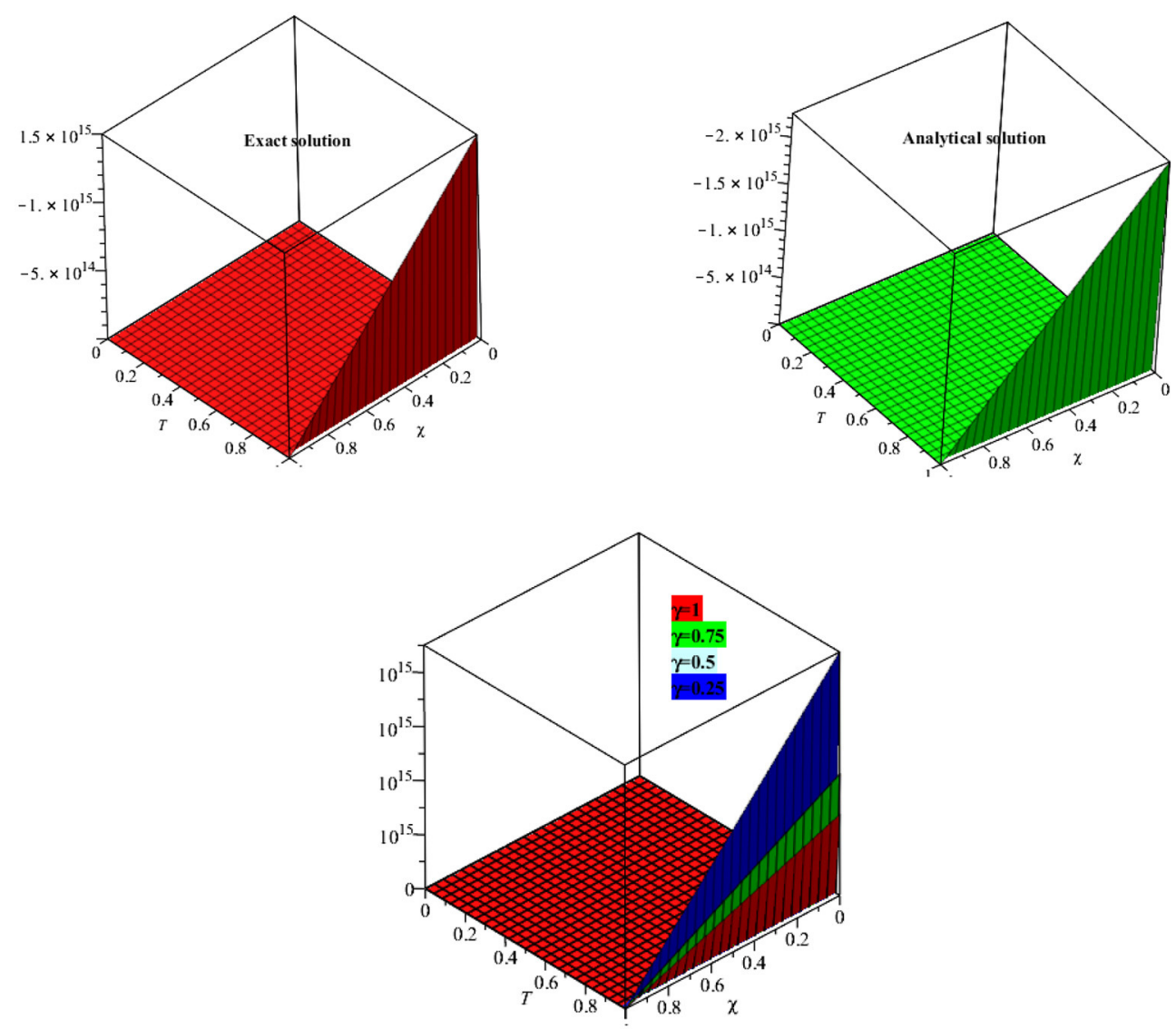

FIGURE 3 | Represents the exact and analytical solution of example 4.2. 

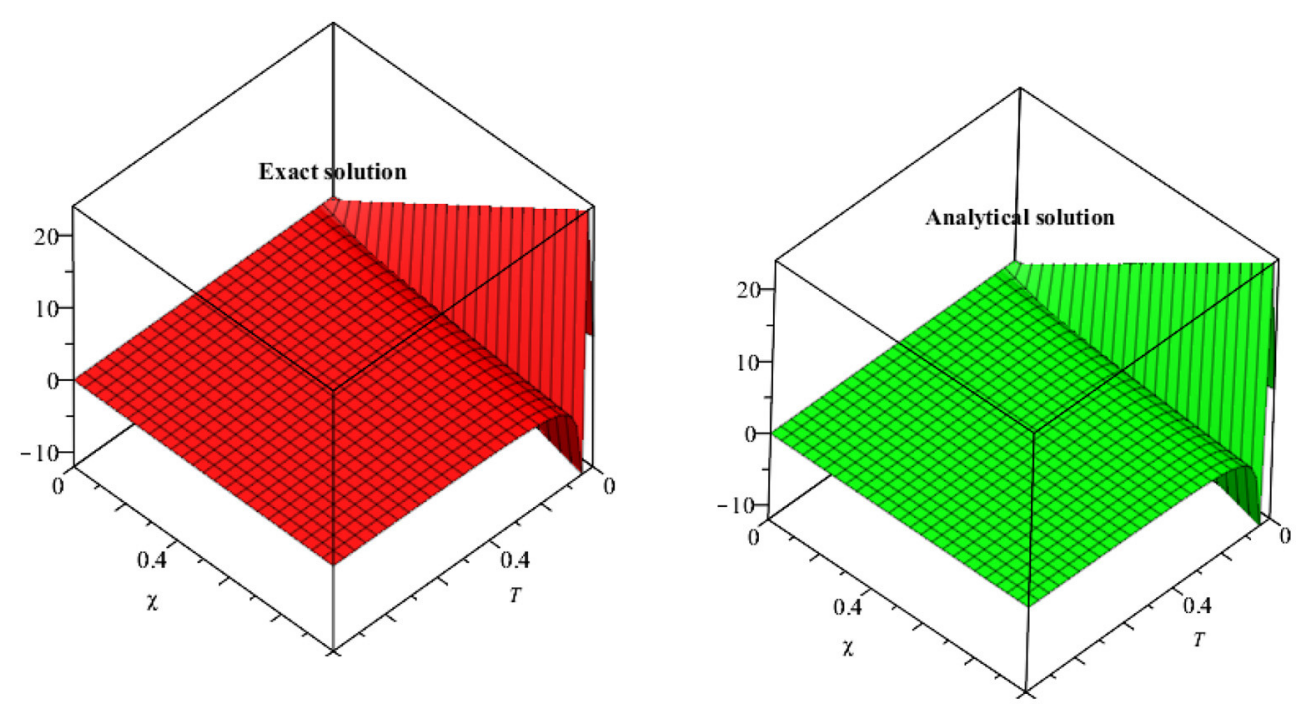

FIGURE 4 | Represents the exact and analytical solution of example 4.3.
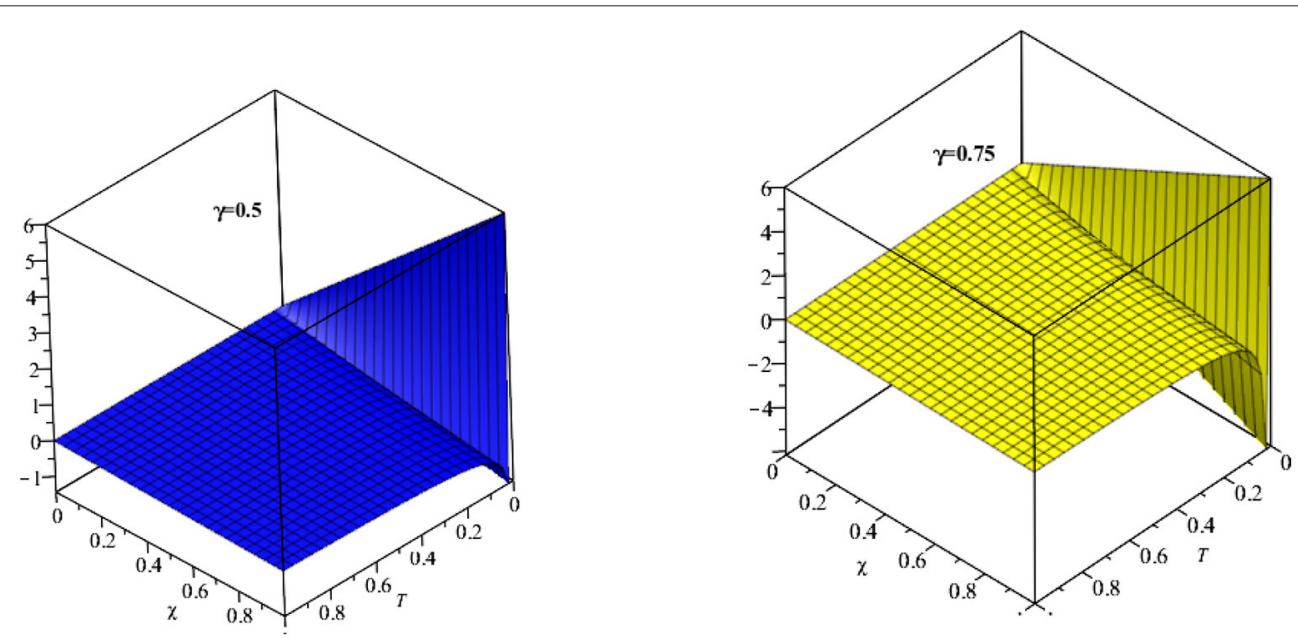

FIGURE 5 | Represents the solution of example 4.3 at $\gamma=0.75, \gamma=0.5$.

\section{RESULTS AND DISCUSSION}

In Figure 1, the exact and analytical solutions of example 4.1 are presented. The solution-graph have confirmed that the obtained results are in good contact with the exact solutions of example 4.1. In Figure 2, the fractional-order solutions are calculated at fractional-order $\gamma=1,0.9,0.7$, and 0.5 . The solutions graphs are expressed in both two and three dimensions. The convergence phenomena can be observed from Figure 2. The similar implementation and results can be seen in Figures 3-7 for example 4.3 and 4.4 also. In Table 1, the results of MDM are compared with the results of HPTM which provide identical results. It is observed that the proposed method has the sufficient accuracy and rate of convergence to the exact solutions of the problems. It is also investigated that the proposed method provided the simple and straightforward implementation for all examples 1, 2, 3, and 4. These investigations of results have confirmed that the present method can be extended to other fractional-order problems arising in science and engineering.

\section{CONCLUSION}

The proposed method is considered to be one of the pre-eminent and new analytical technique, to solve fractional order partial differential equation. In current research article, the proposed method is applied to solve fractional-order kortewege-De Vries equations. The current method is constructed by using Mohand transformation along with Adomian decomposition method. The new hybrid method is very useful to handle the analytical 

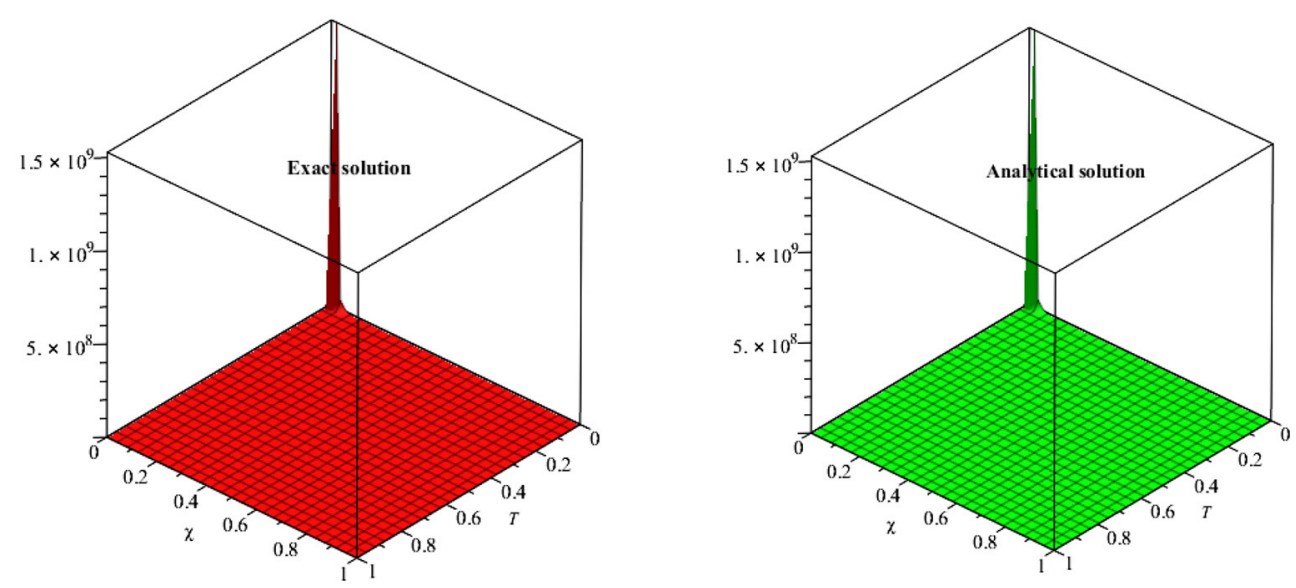

FIGURE 6 | Represents the exact and analytical solution of example 4.4.
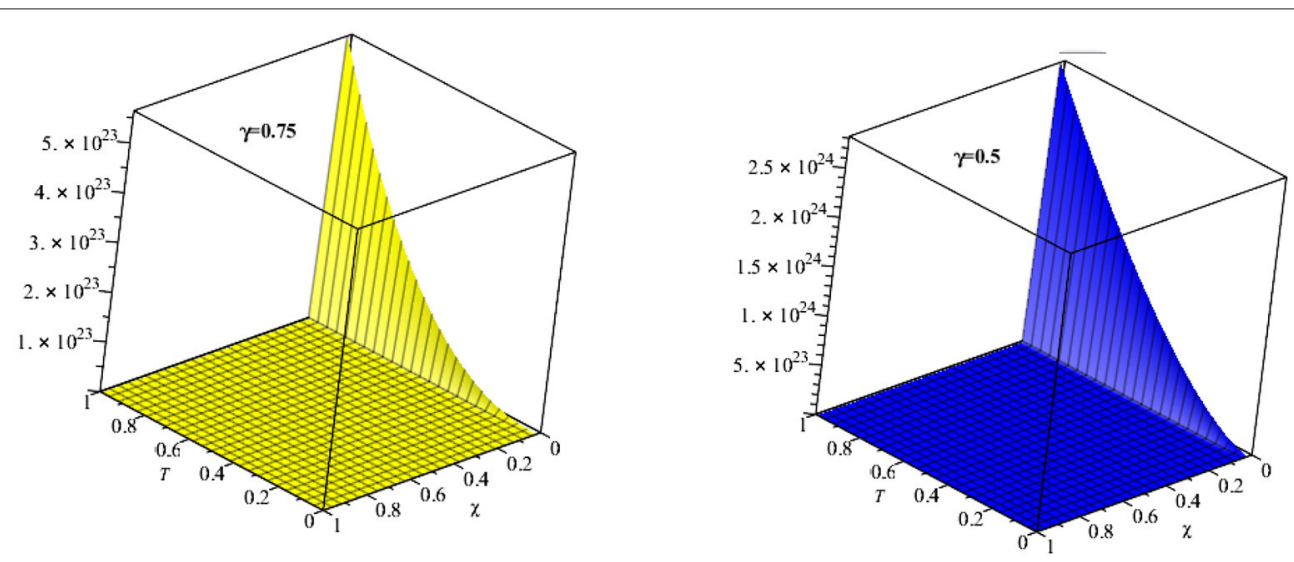

FIGURE 7 | Represents the solution of example 4.4 at $\gamma=0.75, \gamma=0.5$.

TABLE 1 | Comparison of MDM and HPTM [49] of example 1 at $\Im=0.5$.

\begin{tabular}{lccccc}
\hline & MDM & MDM & MDM & $\begin{array}{c}\text { Absolute } \\
\text { error }\end{array}$ & $\begin{array}{c}\text { Absolute } \\
\text { error }\end{array}$ \\
\cline { 2 - 6 }$\chi$ & $\boldsymbol{\gamma}=\mathbf{0 . 5 5}$ & $\boldsymbol{\gamma}=\mathbf{0 . 7 5}$ & $\boldsymbol{\gamma}=\mathbf{1}$ & $\begin{array}{c}\text { HPTM } \\
(\boldsymbol{\gamma}=\mathbf{1})\end{array}$ & $\begin{array}{c}\text { MDM } \\
(\boldsymbol{\gamma}=\mathbf{1})\end{array}$ \\
\hline 0.1 & 0.0712628292 & 0.0893256192 & 0.0970873 & $7.86 \mathrm{E}-08$ & $7.85 \mathrm{E}-08$ \\
0.2 & 0.1425256585 & 0.1786512385 & 0.1941746 & $1.57 \mathrm{E}-07$ & $1.56 \mathrm{E}-07$ \\
0.3 & 0.2137884877 & 0.2679768577 & 0.2912619 & $2.35 \mathrm{E}-07$ & $2.35 \mathrm{E}-07$ \\
0.4 & 0.2850513169 & 0.3573024770 & 0.3883492 & $3.14 \mathrm{E}-07$ & $3.14 \mathrm{E}-07$ \\
0.5 & 0.3563141462 & 0.4466280962 & 0.4854365 & $3.93 \mathrm{E}-07$ & $3.93 \mathrm{E}-07$ \\
0.6 & 0.4275769754 & 0.5359537154 & 0.5825238 & $4.71 \mathrm{E}-07$ & $4.71 \mathrm{E}-07$ \\
0.7 & 0.4988398046 & 0.6252793347 & 0.6796111 & $5.50 \mathrm{E}-07$ & $5.50 \mathrm{E}-07$ \\
0.8 & 0.5701026338 & 0.7146049539 & 0.7766984 & $6.29 \mathrm{E}-07$ & $6.29 \mathrm{E}-07$ \\
0.9 & 0.6413654631 & 0.8039305732 & 0.8737857 & $7.07 \mathrm{E}-07$ & $7.07 \mathrm{E}-07$ \\
1 & 0.7126282923 & 0.8932561924 & 0.9708730 & $7.86 \mathrm{E}-07$ & $7.86 \mathrm{E}-07$
\end{tabular}

solutions of fractional-order partial differential equations. To verify, the validity of the suggested method some numerical examples of time fractional third order $\mathrm{KdV}$ equations are considered to solve it analytically. The solution graphs have confirmed the validity and reliability of the suggested method toward the solutions of other fractional-order non-linear partial differential equations.

\section{DATA AVAILABILITY STATEMENT}

The datasets generated for this study are available on request to the corresponding author.

\section{AUTHOR CONTRIBUTIONS}

HK, RS, and UF has the primary contribution to produce this manuscript. PK has provided the financial support to publish this article. DB and MA have provided their expert opinion and writing draft of the paper.

\section{FUNDING}

The research grant for this manuscript was provided by $\mathrm{PK}$, Center of Excellence in Theoretical and Computational Science 
(TaCS-CoE) and KMUTTFixed Point Research Laboratory, Room SCL 802 Fixed Point Laboratory, Science Laboratory Building, Departments of Mathematics, Faculty of Science,
King Mongkut's University of Technology Thonburi (KMUTT), 126 Pracha-Uthit Road, Bang Mod, Thrung Khru, Bangkok 10140, Thailand.

\section{REFERENCES}

1. Miura RM, editor. Bäcklund transformations, the inverse scattering method, solitons, and their applications: In: Proceedings of the NSF Research Workshop on Contact Transformations. Nashville, TN: Springer (2006).

2. Asif M, Ul Haq S, Islam S, Abdullah Alkanhal T, Khan ZA, Khan I, et al. Unsteady flow of fractional fluid between two parallel walls with arbitrary wall shear stress using caputofabrizio derivative. Symmetry. (2019) 11:449. doi: 10.3390/sym11040449

3. Whitham GB. Linear and nonlinear waves, Vol. 42. John Wiley \& Sons (2011). doi: 10.1002/9781118032954

4. Ali F, Murtaza S, Khan I, Sheikh NA, Nisar KS. AtanganaBaleanu fractional model for the flow of Jeffrey nanofluid with diffusion-thermo effects: applications in engine oil. Adv Diff Equat. (2019) 2019:346. doi: 10.1186/s13662-019-2222-1

5. Lund LA, Omar Z, Alharbi SO, Khan I, Nisar KS. Numerical investigation of multiple solutions for caputo fractional-order-two dimensional magnetohydrodynamic unsteady flow of generalized viscous fluid over a shrinking sheet using the Adams-type predictor-corrector method. Coatings. (2019) 9:548. doi: 10.3390/coatings9090548

6. Kaya D, Aassila M. An application for a generalized KdV equation by the decomposition method. Phys Lett A. (2002) 299:201-6. doi: 10.1016/S0375-9601(02)00652-7

7. Drazin PG, Robin SJ. Solitons: An Introduction. Vol. 2. Cambridge: Cambridge University Press (1989).

8. Saucez P, Vande Wouwer A, Schiesser WE. An adaptive method of lines solution of the Korteweg-de Vries equation. Comput Math Appl. (1998) 35:13-25. doi: 10.1016/S0898-1221(98)00093-5

9. Abassy TA, El-Tawil MA, El-Zoheiry H. Exact solutions of some nonlinear partial differential equations using the variational iteration method linked with Laplace transforms and the Pad technique. Comput Math Appl. (2007) 54:940-54. doi: 10.1016/j.camwa.2006.12.067

10. Kangalgil F, Ayaz F. Solitary wave solutions for the KdV and mKdV equations by differential transform method. Chaos Solit Fract. (2009) 41:464-72. doi: 10.1016/j.chaos.2008.02.009

11. Ganji DD, Mirmohammadsadeghi SE, Safari M. Application fo He's variational iteration method and Adomian's decomposition method to prochhammer-chree equation. Int J Mod Phys B. (2009) 23:435-46. doi: 10.1142/S0217979209049656

12. Marin M. On the domain of influence in thermoelasticity of bodies with voids. Arch Math (Brno). (1997) 33:301-8.

13. Shah R, Khan H, Arif M, Kumam P. Application of Laplace Adomian decomposition method for the analytical solution of third-order dispersive fractional partial differential equations. Entropy. (2019) 21:335. doi: 10.3390/e21040335

14. Othman MIA, Marin M. Effect of thermal loading due to laser pulse on thermoelastic porous medium under GN theory. Results Phys. (2017) 7:386372. doi: 10.1016/j.rinp.2017.10.012

15. Momani S, Odibat Z, Alawneh A. Variational iteration method for solving the space and time fractional KdV equation. Numer Methods Part Diff Equat. (2008) 24:262-71. doi: 10.1002/num.20247

16. Wang Q. Homotopy perturbation method for fractional KdV equation. Appl Math Comput. (2007) 190:1795-802. doi: 10.1016/j.amc.2007. 02.065

17. Wang Q. Homotopy perturbation method for fractional KdV-Burgers equation. Chaos Solit Fract. (2008) 35:843-50. doi: 10.1016/j.chaos.2006.05.074

18. Momani S. An explicit and numerical solutions of the fractional KdV equation. Math Comput Simulat. (2005) 70:110-8. doi: 10.1016/j.matcom.2005.05.001

19. Song L, Zhang H. Application of homotopy analysis method to fractional KdVBurgersKuramoto equation. Phys Lett A. (2007) 367:88-94. doi: 10.1016/j.physleta.2007.02.083

20. Shah R, Khan H, Farooq U, Baleanu D, Kumam P, Arif M. A new analytical technique to solve system of fractional-order partial differential equations. IEEE Access. (2019) 7:150037-50. doi: 10.1109/ACCESS.2019.2946946

21. Jeffrey A. Applied Partial Differential Equations: An Introduction. San Diego, CA: Academic Press (2003).

22. Satsuma J, Kaup DJ. A Bcklund transformation for a higher order Kortewegde Vries equation. J Phys Soc Jpn. (1977) 43:692-7. doi: 10.1143/JPSJ. 43.692

23. Grses M, Karasu A. Variable coefficient third order Kortewegde Vries type of equations. J Math Phys. (1995) 36:3485-91. doi: 10.1063/1.530974

24. Chavan SS, Panchal MM. Solution of third order Korteweg-De Vries equation by homotopy perturbation method using Elzaki transform. Int J Res Appl Sci Eng Technol. (2014) 2:366-9.

25. Debnath L, Bhatta D. Integral Transforms and Their Applications. New York, NY: Chapman and Hall/CRC Press (2014).

26. Mahgoub, MAM. The new integral transform "Mahgoub Transform". Adv Theor Appl Math. (2016) 11:391-8.

27. Abdelilah K, Hassan S. The new integral transform "Kamal Transform". Adv Theor Appl Math. (2016) 11:451-8.

28. Elzaki TM. The new integral transform "Elzaki transform”. Glob J Pure Appl Math. (2011) 7:57-64.

29. Aboodh KS. The new integral transform “Aboodh Transform”. Glob J Pure Appl Math. (2013) 9:35-43.

30. Mohand M, Mahgoub A. The new integral transform Mohand Transform. Adv Theor Appl Math. (2017) 12:113-20.

31. Watugala GK. Sumudu transform: a new integral transform to solve differential equations and control engineering problems. Integr Educ. (1993) 24:35-43. doi: 10.1080/0020739930240105

32. Khan H, Shah R, Baleanu D, Kumam P, Arif M. Analytical solution of fractional-order hyperbolic telegraph equation, using natural transform decomposition method. Electronics. (2019) 8:1015. doi: 10.3390/electronics8091015

33. Eltayeb H, Klman A. A note on the Sumudu transforms and differential equations. Appl Math Sci. (2010) 4:1089-98.

34. Aggarwal S, Singh GP. Shehu Transform of Error Function (probability integral). Int J Res Advent Technol. (2019) 7:54-60. doi: 10.32622/ijrat.76201932

35. Shah R, Khan H, Baleanu D, Kumam P, Arif M. A novel method for the analytical solution of fractional ZakharovKuznetsov equations. Adv Diff Equat. (2019) 2019:1-14. doi: 10.1186/s13662-019-2441-5

36. Aggarwal S, Gupta AR. Solution of linear Volterra integro-differential equations of second kind using Kamal transform. J Emerg Technol Innovat Res. (2019) 6:741-7.

37. Kumar PS, Viswanathan A. Applications of Mohand transform to mechanics and electrical circuit problems. Int J Res Adv Technol. (2018) 6:2838-40.

38. Aggarwal S, Chauhan R. A comparative study of Mohand and Aboodh transforms. Int J Res Adv Technol. (2019) 7:520-9. doi: 10.32622/ijrat.712019107

39. Shah R, Khan H, Baleanu D. Fractional WhithamBroerKaup Equations within Modified Analytical Approaches. Axioms. (2019) 8:125. doi: 10.3390/axioms 8040125

40. Aggarwal S, Sharma SD. A comparative study of Mohand and Sumudu transforms. J Emerg Technol Innovat Res. (2019) 6:145-53.

41. Aggarwal S. A comparative study of Mohand and Mahgoub transforms. Stat. (2019) 4:1-7.

42. Aggarwal S, Raman C, Nidhi S. Mohand transform of Bessels functions. Int $J$ Res Adv Technol. (2018) 6:3034-8. 
43. Aggarwal S, Sharma N, Chauhan RŚolution of linear Volterra integral equations of second kind using Mohand transform. Int J Res Adv Technol. (2018) 6:3098-102.

44. Srivastava HM, Shah R, Khan H, Arif M. Some analytical and numerical investigation of a family of fractional order Helmholtz equations in two space dimensions. Math Methods Appl Sci. (2020) 43:199-212. doi: $10.1002 / \mathrm{mma} .5846$

45. Mohand M, Mahgoub A. The new integral transform Mohand Transform. Adv Theor Appl Math. (2017) 12:113-20.

46. Aggarwal S, Mishra R, Chaudhary A. A comparative study of Mohand and Elzaki transforms. Glob J Eng Sci Res. (2019) 6: 203-13.

47. Hilfer R, editor. Applications of Fractional Calculus in Physics. Singapore: World Scientific (2000).

48. Shah K, Khalil H, Ali Khan R. Analytical solutions of fractional order diffusion equations by natural transform method. Iran $J$ Sci Technol Trans A. (2018) 42:1479-90. doi: 10.1007/s40995-0160136-2
49. Eljaily MH, Elzaki TM. Homotopy perturbation transform method for solving korteweg-devries (kdv) equation. Pure Appl Math J. (2015) 4:264-8. doi: 10.11648/j.pamj.20150406.17

50. Sedeeg AKH. Homotopy perturbation transform method for solving third order Korteweg De-Vries (KDV) Equation. AJAM. (2016) 4:247-51. doi: 10.11648/j.ajam.20160405.16

Conflict of Interest: The authors declare that the research was conducted in the absence of any commercial or financial relationships that could be construed as a potential conflict of interest.

Copyright $\odot 2020$ Shah, Farooq, Khan, Baleanu, Kumam and Arif. This is an openaccess article distributed under the terms of the Creative Commons Attribution License (CC BY). The use, distribution or reproduction in other forums is permitted, provided the original author(s) and the copyright owner(s) are credited and that the original publication in this journal is cited, in accordance with accepted academic practice. No use, distribution or reproduction is permitted which does not comply with these terms. 